\title{
La agricultura en APEC
}

DOI: $10.32870 /$ mycp.v4i13.135

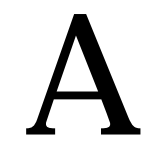
medida que las economías avanzan en su proceso de desarrollo, la participación relativa de la agricultura en el producto interno bruto (PIB) tiende a descender. Este hecho no disminuye la importancia estratégica de la producción, comercialización, procesamiento y consumo de los alimentos; por el contrario, el impacto social, político y cultural de las decisiones económicas, con respecto al sector agrícola, hace que éstas sean extremadamente cuidadas en todos los niveles: internacional, regional y nacional.

Precisamente, la dificultad de abordar el tema agrícola en los debates internacionales, ocasionó que haya sido hasta la Ronda de Uruguay (RU) del GATT (1989-1994) cuando la agricultura fue incluida por primera vez en las negociaciones de este organismo. En noviembre de 2000 la agricultura volvió a constituirse en un tema de controversia en la reunión preparatoria para la nueva ronda de negociaciones de la OMC. Sea por razones de seguridad nacional, por proteger a ciertos grupos de productores o por razones culturales, lo cierto es que la agricultura ha constituido siempre uno de los sectores más protegidos.

Con el nuevo proceso de globalización, la mayoría de los países en desarrollo han seguido políticas unilaterales de apertura, incluyendo a sus sectores agrícolas, en los que las exportaciones constituyen una fuente importante de divisas. Sin embargo, los países desarrollados no han respondido cabalmente a

* Investigadores del Departamento de Estudios del Pacífico de la Universidad de Guadalajara.

ORCID http://orcid.org/0000-0003-4926-0594

ORCID http://orcid.org/0000-0002-9145-5865
Melba E. Falck y Roberto Hernández Hernández*

los compromisos de liberalización agrícola contraídos en la RU y lo que repercute negativamente en el desenvolvimiento exportador de aquellos, por estas razones, el tema de la agricultura en APEC constituye actualmente uno de los asuntos prioritarios para las economías de la región.

\section{La agricultura en la región}

La característica principal de la agricultura, entre los países de APEC, es la desigualdad, manifiesta en la dotación relativa de recursos naturales y en las políticas agrícolas de sus gobiernos. También son muy marcadas las diferencias, en cuanto a los modos de producción, comercialización e ingresos que perciben los productores agrícolas.

En términos de dotación de recursos agrícolas, Estados Unidos, Australia y Nueva Zelanda presentan elevados niveles de tierra agrícola por trabajador: 159 hectáreas para Estados Unidos y 90 para los otros dos países. Le siguen México y Canadá con 10 y 13 hectáreas respectivamente. Los países restantes, todos asiáticos, tienen una fuerte escasez, en todos los casos con superficies inferiores a una hectárea por trabajador o familia, de ahí el intensivo uso de la tierra.

Los niveles de producción agrícola son marcadamente dispares en la región; sólo dos países (China y Estados Unidos) concentran el 71.2 por ciento de la producción de cereales, el 68.8 por ciento de oleaginosas y el 77.3 por ciento de vegetales; algunos países más reportan 
niveles medios de producción en cereales, como Rusia y Canadá (5.3 por ciento cada uno), Indonesia (4.3 por ciento) y Australia (3.4 por ciento); en el resto de las economías la producción es poco significativa para la región, no faltan aquellas donde la producción es prácticamente de cero. Este mismo patrón, aunque con porcentajes diferentes, lo encontramos en tubérculos, oleaginosas vegetales y frutas (cuadro 1).

En países como Vietnam, China, Tailandia y Filipinas, el sector agrícola todavía emplea más de la mitad de la fuerza de trabajo; en México y Malasia cerca de una cuarta parte de la mano de obra se encuentra ocupada en el sector. Considerando esos elevados niveles de ocupación agrícola y la contribución de la agricultura en la economía, los niveles de productividad de la mano de obra agrícola son muy bajos. En el caso de México, por ejemplo, aún cuando el sector emplea 28 por ciento de la mano de obra, únicamente contribuye con 8 por ciento al PIB. Esos bajos niveles de productividad se reflejan en un ingreso per cápita rural por debajo de la media nacional.

En el otro extremo están los países en los cuales el sector agrícola presenta elevados niveles de productividad como Australia, Canadá, Estados Unidos y Nueva Zelanda. Con excepción de éste en el cual la agricultura tiene una participación muy alta para un país desarrollado, la contribución del sector a la economía en estos países oscila entre 2 y tres por ciento, y ocupa entre 3 y cinco por ciento de la fuerza de trabajo. Todos estos países tienen

\section{Cuadro 1}

APEC: producción agrícola por grupos de bienes, 1999 (miles de toneladas métricas)

\begin{tabular}{|c|c|c|c|c|c|c|c|c|c|c|}
\hline & \multicolumn{2}{|c|}{ cereales* } & \multicolumn{2}{|c|}{$\begin{array}{c}\text { raíces y } \\
\text { tubérculos }\end{array}$} & \multicolumn{2}{|c|}{$\begin{array}{c}\text { semillas } \\
\text { oleaginosas }\end{array}$} & \multicolumn{2}{|c|}{ vegetales } & \multicolumn{2}{|l|}{ frutas } \\
\hline & cantidad & $\%$ & cantidad & $\%$ & cantidad & $\%$ & cantidad & $\%$ & cantidad & $\%$ \\
\hline Australia & 34,677 & 3.4 & 1,333 & 0.4 & 3,706 & 2.0 & 1,799 & 0.5 & 2,918 & 1.8 \\
\hline Brunei & 297 & 0.0 & 2,100 & 0.7 & 135 & 0.1 & 9,200 & 2.4 & 5,640 & 3.4 \\
\hline Canadá & 53,911 & 5.3 & 4,268 & 1.4 & 13,030 & 7.0 & 2,321 & 0.6 & 913 & 0.6 \\
\hline Corea del Sur & 5,280 & 0.5 & 1,106 & 0.4 & 174 & 0.1 & 10,980 & 2.8 & 2,468 & 1.5 \\
\hline Chile & 2,148 & 0.2 & 1,002 & 0.3 & 87 & 0.0 & 2,591 & 0.7 & 4,118 & 2.5 \\
\hline China & 388,458 & 38.4 & 187,545 & 61.1 & 46,435 & 24.8 & 260,081 & 67.2 & 64,832 & 39.4 \\
\hline China Hong Kong & 0 & 0.0 & 0 & 0.0 & 96 & 0.1 & 55 & 0.0 & 4 & 0.0 \\
\hline China Taiwán & 1,514 & 0.1 & 327 & 0.1 & 101 & 0.1 & 3,378 & 0.9 & 2,445 & 1.5 \\
\hline Estados Unidos & 332,444 & 32.8 & 22,249 & 7.3 & 82,329 & 44.0 & 39,053 & 10.1 & 28,090 & 17.1 \\
\hline Filipinas & 12,446 & 1.2 & 2,732 & 0.9 & 5,810 & 3.1 & 4,728 & 1.2 & 9,398 & 5.7 \\
\hline Indonesia & 43,132 & 4.3 & 19,248 & 6.3 & 19,579 & 10.5 & 6,397 & 1.7 & 7,478 & 4.5 \\
\hline Japón & 8,463 & 0.8 & 4,489 & 1.5 & 206 & 0.1 & 12,838 & 3.3 & 4,245 & 2.6 \\
\hline Malasia & 1,415 & 0.1 & 469 & 0.2 & 3,832 & 2.0 & 488 & 0.1 & 1,066 & 0.6 \\
\hline México & 28,293 & 2.8 & 1,597 & 0.5 & 1,893 & 1.0 & 9,641 & 2.5 & 11,390 & 6.9 \\
\hline Nueva Zelanda & 876 & 0.1 & 518 & 0.2 & 5 & 0.0 & 959 & 0.2 & 983 & 0.6 \\
\hline Papua Nva.Guinea & 10 & 0.0 & 1,260 & 0.4 & 895 & 0.5 & 387 & 0.1 & 1,213 & 0.7 \\
\hline Perú & 2,738 & 0.3 & 4,507 & 1.5 & 134 & 0.1 & 1,891 & 0.5 & 3,246 & 2.0 \\
\hline Rusia & 53,697 & 5.3 & 31,344 & 10.2 & 4,695 & 2.5 & 12,689 & 3.3 & 2,354 & 1.4 \\
\hline Singapur & nd & & nd & & nd & & nd & & nd & \\
\hline Tailandia & 20,143 & 2.0 & 16,771 & 5.5 & 2,347 & 1.3 & 2,774 & 0.7 & 7,610 & 4.6 \\
\hline Vietnam & 22,692 & 2.2 & 3,882 & 1.3 & 1,537 & 0.8 & 4,838 & 1.2 & 3,972 & 2.4 \\
\hline Total & $1,012,634$ & 100 & 306,747 & 100 & 187,026 & 1000 & 387,088 & 1000 & 164,383 & 100 \\
\hline
\end{tabular}

* no incluye cebada maltera

FUENTE: FAO, FAOSTAT 
ingresos elevados y disfrutan de abundantes recursos naturales.

Japón, Corea del Sur y Taiwán, que comparten escasez relativa de tierra, emplean a 7,18 y 12 por ciento respectivamente de la fuerza de trabajo en la agricultura, la cual contribuye con 2,7 y 4 por ciento al PIB en cada uno de ellos, reflejando también niveles bajos de rentabilidad económica.

En el periodo 1994-1999 la región participó con 38 por ciento de las exportaciones agroalimentarias del mundo y con el 41 por ciento de las importaciones de las mismas, lo cual indica la importancia de la región en el comercio mundial de alimentos. ${ }^{1}$ El grupo de exportadores netos (Estados Unidos, Australia, Tailandia, Nueva Zelanda, Canadá, Malasia, Chile, Indonesia, Vietnam y Papua Nueva Guinea), contribuyó con el 74.7 por ciento de las e $x$ p o r t a c i o n e s agroalimentarias de la región, con un monto promedio anual de 118,991 millones de dólares (cuadro 2). Estados Unidos figura como líder exportador, al participar con el 37 por ciento de las ventas de la región; seguido de lejos por Australia y Canadá con 9.1 y 8.8 por ciento, respectivamente. Vietnam, aunque tiene una participación menor durante el periodo analizado, se mantuvo como exportador neto, y considerando que se encuentra en periodo de transición, es un logro importante. China también tiene una participación elevada en las exportaciones

Japón
Rusia
Corea del Sur
Hong Kong
Taiwán*
China
México
Filipinas
Singapur
Perú
Brunei

de la región, similar a la de Australia y Canadá (8.4 por ciento) con 13.4 mil millones de dólares, no obstante que en los últimos años este país haya mantenido un saldo deficitario en su balanza agrícola.

En cuanto a la participación de la región los diez países con saldo deficitario en su balanza agrícola, en las importaciones agroalimentarias mundiales, interviene con el 62.5 por ciento de las importaciones regionales, que en total importan un promedio anual de 108 mil millones de dólares. En este caso el líder importador es

Cuadro 2

Comercio agrícola de los países de APEC (miles de dolares)

\begin{tabular}{lrrr}
\hline Países & \multicolumn{3}{c}{ promedio anual 1994-1999 } \\
\cline { 2 - 4 } & & & \\
& & & \\
Exportadores netos & & & \\
& $58,908,044$ & $38,136,125$ & $20,771,919$ \\
Estados Unidos & $14,438,089$ & $2,659,798$ & $11,778,292$ \\
Australia & $7,944,352$ & $2,589,081$ & $5,355,271$ \\
Tailandia & $6,034,196$ & $1,051,550$ & $4,982,646$ \\
Nueva Zelanda & $13,999,778$ & $9,902,391$ & $4,097,386$ \\
Canadá & $7,465,472$ & $3,845,915$ & $3,619,557$ \\
Malasia & $2,491,722$ & $1,160,609$ & $1,331,113$ \\
Chile & $5,412,025$ & $4,415,840$ & 996,184 \\
Indonesia & $1,856,832$ & 939,202 & 917,630 \\
Vietnam & 440,879 & 241,200 & 199,679 \\
Papua Nueva Guinea & & &
\end{tabular}

Importadores netos

$1,637,570$
$1,258,792$
$1,650,481$
$5,241,320$
$4,885,333$
$13,431,610$
$5,922,627$
$1,659,503$
$3,856,728$
642,572
16,922

$38,151,824$

$-36,514,254$

$10,992,191$

$-9,733,399$

$8,674,987$

$-7,024,505$

$10,014,823$

$-4,773,503$

$8,773,000$

$-3,887,667$

$15,120,213$

$-1,688,604$

$7,505,040$

$-1,582,413$

$2,501,255$

$-841,753$

$4,843,285$

$-986,557$

$1,279,138$

$-636,566$

195,562

\footnotetext{
* 1993-1995

FUENTE: FAO, FAOSTAT, Database
} 
Japón, con el 22 por ciento de la región, con un monto promedio de 36.5 mil millones de dólares en el periodo considerado. Le siguen China y Hong Kong, que en conjunto suman 14.5 por ciento (8.7 y 5.8 por ciento, respectivamente). Rusia por su parte participa con el 6.4 por ciento y Corea del Sur y Taiwán con 5 por ciento cada uno. México participa con 4.3 por ciento de las importaciones totales de la región. Estados Unidos es también uno de los principales importadores: participa con 22 por ciento del total, aunque en el periodo considerado mantuvo una balanza comercial muy favorable.

Durante el mismo periodo de 1994 a 1999, la región presentó un saldo en la balanza comercial desfavorable, resultado de un promedio anual de exportaciones de $159.2 \mathrm{mil}$ millones de dólares y de importaciones por un monto de 173 mil millones de dólares, en números redondos. Los principales importadores fueron Japón, Rusia, Corea del Sur, Hong Kong, Taiwán, China y México. Por otra parte, los países con un mayor saldo positivo en su balanza agrícola fueron, Estados Unidos, Australia, Tailandia, Nueva Zelanda y Canadá. La región de APEC, en su conjunto, participa con el 36.6 por ciento de las exportaciones mundiales de productos agrícolas y con el 38.3 por ciento de las importaciones de los mismos bienes (cuadro 2).

Veamos ahora las características básicas de la agricultura de algunos países de la región de $\mathrm{APEC}^{2}$ claves para México, que como es sabido ha volcado su estrategia de desarrollo hacia el exterior. Desde 1985, con su entrada al GATT, liberalizó unilateralmente la economía, y a partir de 1989 emprendió un programa de liberalización del sector agrícola, misma que está conduciendo a una especialización del sector en aquellos productos en los que nuestro país tiene una ventaja comparativa, como frutas y hortalizas. Por otra parte, México ha concentrado sus flujos de comercio exterior agrícola con Estados Unidos, que se aceleraron con la entrada en vigor del Tratado de Libre Comercio (TLCAN) en 1994.

\section{Estados Unidos 3}

Estados Unidos es el principal actor en el comercio agrícola de la región, y por lo tanto su política en este rubro tendrá impactos muy importantes. El nivel de exportaciones agroindustriales de ese país se situó en $60 \mathrm{mil}$ millones de dólares en el periodo 1994-1999, participando con 40 por ciento de las exportaciones de la región. Las importaciones por su parte se mantuvieron en una nivel de 38 mil millones de dólares en promedio (cuadro 2), lo cual significa que participa con una quinta parte de las importaciones de la región.

El principal punto de controversia de la política agrícola de Estados Unidos son los apoyos a los productores y comercializadores que distorsionan el mercado internacional de los bienes agrícolas. Para hacer frente a esta circunstancia y presionar a sus socios comerciales en las negociaciones de la OMC, ha realizado algunas reformas a la misma. La ley agrícola de 1996 (vigente hasta 2002), ha introducido nuevos mecanismos de administración pública de la agricultura para permitir una mayor acción del mercado. En este sentido, por ejemplo, prevé pagos directos a los productores de trigo, granos forrajeros, algodón y arroz, mediante la firma de contratos de flexibilidad productiva, lo que implica la eliminación de los recursos para los precios objetivos y los pagos por deficiencia. Establece la disminución de los precios de apoyo para los productores de lácteos, y elimina la mayoría de los controles sobre superficie que restringían los patrones de siembra. El marco de estos ajustes lo constituye la ley de transición a la agricultura de Mercado 4 .

Aunque el nivel de los apoyos otorgados al sector por parte del gobierno ha tendido a descender a partir de los ochenta, continúa siendo alto. Medido en términos de subsidio equivalente al productor (PSE) es de 17 por ciento; comparado con el 1 y 7 por ciento de Nueva Zelanda y Australia respectivamente ${ }^{5}$. Estados Unidos todavía mantiene restricciones en muchos productos: al azúcar la protege con cuotas, los productos lácteos con precios mínimos 
de apoyo, compras del gobierno, tarifas, cuotas y subsidios a la exportación. Otras industrias pecuarias son protegidas con medidas de frontera, incluyendo un esquema de cuota-tarifa para la carne y subsidios a la exportación para el cerdo, aves y huevos. En 1998, los productos que recibieron un mayor apoyo fueron trigo, maíz, azúcar y leche, con un PSE de 38, 25, 41 y 61 por ciento respectivamente. Si tomamos en cuenta los elevados niveles de participación de Estados Unidos en los mercados internacionales, la mayor apertura de su mercado traería beneficios para otros exportadores netos de Asia Pacífico y México. Nuestro país constituye uno de los principales proveedores de productos alimenticios a Estados Unidos.

\section{Canadá}

La estructura de producción agrícola de Canadá se explica en gran medida por las condiciones climatológicas y el tipo de suelos. El tipo de tierra agrícola varía, significativamente, de una región a otra. En las zonas donde la tierra se destina al cultivo de pastos, maíz, granos, oleaginosas, vegetales y frutas especializadas, las unidades de producción agrícola son “pequeñas”, por lo general, menores a 100 hectáreas. En contraste las praderas, donde los ranchos tienden a especializarse en trigo y canola superan, por lo general, las 200 hectáreas. En Canadá más del 98 por ciento de las unidades de producción son de tipo familiar, en cuanto a la propiedad y su operación.

El sector agrícola de Canadá avanza por el camino de la liberalización. Ello se ha reflejado en la reestructuración de los mecanismos de apoyo agrícola y en muchos casos en el desmantelamiento paulatino de algunos organismos institucionales y la creación, en su lugar, de programas de apoyo que no distorsionan (o distorsionen lo menos posible), los mecanismos de mercado. Esta política, más la liberalización del comercio internacional, son parte esencial del discurso canadiense en los foros internacionales ${ }^{6}$.

Entre los programas de apoyo se encuentran 'el seguro de cosecha', y la 'red de cuentas de estabilización del ingreso'. Otras acciones se concentran en mejorar la estructura contable, en eficientar los servicios a los productores y en reducir los costos de transacción. En granos y oleaginosas, por ejemplo, la eliminación de los subsidios en la transportación por ferrocarril ha obligado a los agricultores a incrementar el valor agregado de sus productos mediante el mejoramiento de sus procesos de producción y comercialización, específicamente, en graduación, selección, limpieza y marca ${ }^{7}$.

La reestructuración productiva y comercial parte de la idea de que Canadá puede beneficiarse de un sistema que enfatice la libre competencia, las ventajas comparativas y la eliminación de algunas formas de intervención gubernamental. También incluye, como afirma Found ${ }^{8}$, convencer a los gobiernos de otros países sobre la conveniencia de reducir los subsidios a los productores. No obstante, el tipo de cultivo también ha estado en función de políticas específicas llevadas a cabo por los consejos de comercialización agrícola (tales como el de la leche), apoyados por los gobiernos federal y provincial, que controlan los niveles de producción para que la oferta se adecue a la demanda, aunque los precios no sean competitivos a nivel internacional ${ }^{9}$. El sector agroalimentario de Canadá también es apoyado por avanzados centros de investigación federales, provinciales y universitarios.

Dada la gran variedad de producción agrícola, Canadá es más que autosuficiente en la producción de la mayoría de los bienes agrícolas. Es altamente superavitario en cereales, linaza y canola, pero es significativamente deficitario en soya, frutas y vegetales así como en carne de carnero y cordero.

\section{China}

Por el tamaño de su población, cercana a los 1,300 millones de habitantes, así como por las elevadas tasas de crecimiento que experimentó a partir de los ochenta, China constituye uno de los actores centrales en la producción y el comercio de productos agrícolas. El 
comportamiento de la producción y de la demanda de alimentos en este país puede modificar sustancialmente los precios internacionales de los alimentos dependiendo de su participación en el mercado mundial.

Con 7 por ciento de la tierra cultivable a nivel mundial, China tiene el reto formidable de alimentar al 20 por ciento de la población del mundo. Actualmente el sector agrícola participa con el 30 por ciento del PIB y emplea 74 por ciento de la fuerza de trabajo. Aunque la transformación de este sector en los últimos veinte años ha sido muy radical, se esperan ajustes posteriores, como resultado de las tasas de crecimiento económico y la mayor incorporación de la economía al proceso de globalización. Este reto que tiene China de alimentar a una población urbana en constante crecimiento y con mayores niveles de ingreso, le obliga a poner énfasis en la productividad agrícola. Específicamente, sobre el punto de la productividad, los datos estadísticos más recientes proporcionados por la FAO nos permiten afirmar que este país cuenta aún con márgenes sustanciales, tanto de frontera agrícola, como tecnológica, sobre todo en productos básicos ${ }^{10}$.

A finales de la década de los setenta, China puso en práctica su política de "puertas abiertas". El sector rural fue el primero en aplicar una profunda transformación estructural, que cambiaría las formas de producción y distribución de alimentos. Las comunas populares fueron formalmente eliminadas y sustituidas por millones de unidades familiares de producción (sistema de responsabilidad de contrato familiar). El resultado fue un incremento sustancial de la producción agrícola. Los mecanismos de comercialización de alimentos, que el gobierno directamente controlaba en todos los eslabones de la cadena alimentaria, fueron modificados para permitir la acción de las fuerzas del mercado y la participación directa de los agentes particulares -nacionales y extranjeras- en estas actividades; en este sentido, los sistemas de racionamiento y de control de precios de los alimentos fueron eliminados.
Hasta ahora, China ha sido capaz de mantener niveles de producción agrícola satisfactorios en función de la demanda de su población, al grado de sostener un nivel de autosuficiencia alimentaria cercana al cien por ciento. El estancamiento en la producción de granos, que se agudizó en 1995, propició que los líderes chinos pusieran énfasis en el control de mercado para incrementar la producción de estos y elevar los niveles de autosuficiencia en la producción de alimentos. Por medio del "sistema de responsabilidad de bolsa de granos de los gobernadores", se han diseñado estrategias y realizado acciones para estimular a los productores a sembrar más productos básicos. Con esta política se ha restringido el uso alternativo de la tierra en los cultivos de mayor valor agregado a favor de los cereales.

Como parte de las negociaciones para ingresar a la OMC, China ha eliminado muchas de sus barreras al comercio, diminuido aranceles y ampliado sus cuotas de importación de alimentos; su participación formal en este organismo le obligará a realizar ajustes subsiguientes que impactarán aún más sus sistemas de producción y distribución de estos bienes. Por ejemplo, no podrá fijar precios a los productos agrícolas por arriba de los internacionales. Pero el comercio es una avenida de doble sentido. Ello significa que China tendrá un mayor acceso a los mercados internacionales y podrá incrementar sus exportaciones de bienes industriales como calzado, textiles, juguetes y enseres domésticos. Un mayor ingreso de divisas y el mantenimiento de altas tasas de crecimiento económico, podrían impulsar la importación de alimentos. Los países más beneficiados serían además de Estados Unidos, los países del sudeste asiático como Tailandia, Filipinas, Indonesia y Malasia (ASEAN-4) ${ }^{11}$.

\section{Vietnam ${ }^{12}$}

Vietnam comparte con China una estrategia de transición de una economía socialista a una de mercado. Al igual que China, el sector agrícola participa con cerca de 30 por ciento del PIB, empleando el 70 por ciento de su fuerza de trabajo en esas actividades. Lo que indica 
todavía un muy bajo nivel de productividad de esta mano de obra.

El crecimiento de la producción agrícola en Vietnam ha mostrado elevadas tasas en los noventa, de 5.3 por ciento, mientras la economía lo hacía a un 8.3 por ciento, resultado de su nueva estrategia de desarrollo a fines de los setenta, en que su mayor éxito lo constituyó precisamente el sector agrícola, pasando de ser un país deficitario en alimentos a uno superavitario. En suma, Vietnam se está convirtiendo en un competidor importante en este mercado. Sin embargo, los beneficios del crecimiento agrícola no se han repartido equitativamente y en el campo vietnamita se incrementan las disparidades en el ingreso.

\section{ASEAN $-4^{13}$}

En cuanto a su participación en el comercio agrícola de la región, el nivel de exportaciones del grupo alcanzó un monto de 22.5 mil millones de dólares en el periodo 1994-1999, con una participación de 14.1 por ciento en el total de la región. Las importaciones por su parte, se mantuvieron en un nivel promedio de $12.5 \mathrm{mil}$ millones de dólares para los cuatro países en conjunto, equivalentes al 7.5 por ciento de las importaciones de la región (cuadro 2).

Además de poseer una abundancia de recursos naturales y mano de obra, con respecto al grupo de importadores agrícolas netos, los países de ASEAN-4 han compartido experiencias similares en sus estrategias de desarrollo. Todos han seguido, a partir de los sesenta o setenta, un modelo de sustitución de importaciones que por su naturaleza tendió a introducir un sesgo antiagrícola en sus economías.

Con sus especificidades, en cada país se comienza a reconocer la falta de una política de desarrollo agrícola, aunque ésta hubiera estado contenida en los planes de desarrollo empleados por casi todas las economías. Así, en los noventa en Malasia, se planteó la diversificación agrícola: horizontalmente en cultivos industriales, alimenticios y hortícolas, y verticalmente convertir a Malasia en el centro de procesamiento de alimentos de ASEAN. No obstante, uno de los mayores problemas que enfrenta Malasia es la escasez de mano de obra, pues la producción hortícola y el procesamiento de alimentos son actividades intensivas de esa característica. Actualmente Malasia permite la contratación de fuerza de trabajo extranjera procedente de Indonesia y Filipinas. Con la excepción de frutas, en el periodo 1990-1999, Malasia presentó bajas tasas de autosuficiencia en cereales, vegetales y oleaginosas (cuadro 3).

Tailandia por su parte, desde los sesenta es un país superavitario en el comercio de alimentos y sigue manteniendo altos niveles de autosuficiencia en las cinco categorías de productos consideradas. Sin embargo, Tailandia parece haber llegado a una encrucijada, ya que con el patrón de desarrollo agrícola adoptado, sobre la base de la práctica del monocultivo, un alto porcentaje de las tierras se encuentran erosionadas y cerca de la mitad de los bosques ha sido reclamada por la agricultura. El problema actual para Tailandia es cómo hacer compatible el desarrollo de la agroindustria con una agricultura sustentable menos agresiva al medio ambiente. La exportación agroindustrial en Tailandia ha estado creciendo rápidamente en productos como frutas y vegetales enlatados, tales como piña y maíz dulce. Dos terceras partes de estas exportaciones van a países desarrollados, Estados Unidos, Europa, y el resto a Asia. La exportación de mango y toronja también es importante. Cabe hacer notar que la tecnología utilizada en la producción de frutas en Tailandia es de las más avanzadas en el mundo actualmente. La consolidación de las redes de transporte en el territorio tailandés también ha favorecido el desarrollo del sector.

Indonesia, que comenzó su estrategia de promoción de exportaciones en los ochenta, logró alcanzar la autosuficiencia en arroz al tiempo que expandía la producción de cultivos comerciales como el aceite de palma, cacao, té y caucho. En cacao logró situarse como el mayor 
exportador mundial. El incremento de la producción en Indonesia se logró con la expansión de la frontera agrícola, ya que excepto el arroz, la productividad agrícola es baja en relación con los otros tres países. En el campo indonesio coexisten las grandes plantaciones estatales con las pequeñas unidades productoras. Aparte de una baja productividad en el sector agrícola, Indonesia no ha sido capaz de aprovechar sus ventajas comparativas en frutas y vegetales.

De los cuatro países, Filipinas es el que muestra las menores tasas de crecimiento y el menor nivel de ingreso per cápita. Aunque la agricultura mostró un buen desempeño en el periodo 1965-1980, a partir de este año hasta 1995 el sector sólo ha crecido en promedio 1.3 por ciento anual. En el primer periodo la tasa de expansión de 4.3 por ciento mostrada por el sector se debió más que todo al crecimiento en maíz, plátanos, piñas y café. Sin embargo, a pesar de la revolución verde, el arroz no fue el cultivo que más contribuyó al crecimiento.

En los ochenta ya no se expandió la frontera agrícola; la lenta aplicación de la reforma agraria de 1988 ha introdujo incertidumbre en el campo, desalentando la inversión y apresurando la venta de tierras agrícolas a usos no agrícolas. En el periodo 19911997 Filipinas importó 15 por ciento de los cereales que consume, aunque es autosuficiente en vegetales y frutas.

En resumen, estas cuatro economías enfrentan todavía problemas serios en sus respectivos sectores agrícolas. El agotamiento de la frontera agrícola hace todavía más importante una mejora en la productividad sujeta a la restricción de desarrollar una agricultura menos agresiva con el medio ambiente, que a su vez impondrá límites al crecimiento del producto. Con la excepción de Malasia, los sectores industriales de estos países deben crecer lo suficiente para absorber la mano de obra excedente en el sector agrícola una vez que este incremente su productividad.
Superados estos problemas, es grande el potencial de crecimiento del sector. Si las políticas de liberalización en sus vecinos asiáticos, (Japón, Corea, Taiwán y China), se llevan a cabo, tales economías se beneficiarían en gran medida.

\section{Autosuficiencia alimentaria}

El nivel de autosuficiencia alimentaria permite apreciar, de manera general, la estructura y la tendencia del comercio exterior agrícola de los países. En el caso de APEC encontramos una tendencia hacia la disminución por parte de los países menos desarrollados en el nivel de autosuficiencia alimentaria de productos básicos (cereales, tubérculos y oleaginosas), compensada, en la mayoría de los casos, con la producción de frutas y vegetales.

En cuanto al grado de autosuficiencia alimentaria por países, la región presenta el siguiente perfil: las economías que presentan una menor autosuficiencia alimentaria en cereales son: Japón, con una tasa de 25 por ciento, es decir importa 75 por ciento de su consumo interno de cereales, seguido por Malasia con tasa del 29 por ciento y Corea del Sur con 32 por ciento. Le sigue, en Latinoamérica, Perú con un nivel de autosuficiencia de 43 por ciento. México y Nueva Zelanda importan 20 por ciento de los cereales que consumen; Rusia e Indonesia el 10 por ciento. China por su parte es prácticamente autosuficiente en cereales, mientras que Australia, Canadá, Estados Unidos, Tailandia y Vietnam son abastecedores mundiales de este grupo de alimentos. Paradójicamente, países como Japón y Corea del Sur que han sido tradicionalmente proteccionistas con sus sectores agrícolas, son los que presentan las menores tasas de autosuficiencia. En el caso de raíces y tubérculos, con excepción de Brunei, Corea del Sur, Japón, Malasia y México, el resto de los países de la región presentan niveles de autosuficiencia o cercanos a ella. Este no es el caso de oleaginosas, donde la mayoría de los países presentan marcados déficits, que se 
Cuadro 3

APEC: nivel de autosuficienica alimentaria por producto y país, 1990-1997 y 1999

\begin{tabular}{|c|c|c|c|c|c|c|c|c|}
\hline & \multicolumn{2}{|c|}{ cereales $^{*}$} & \multicolumn{2}{|c|}{ raíces y tubérculos } & \multicolumn{2}{|c|}{ oleaginosas } & \multirow{2}{*}{$\begin{array}{r}\text { vegetales } \\
1999 \\
\end{array}$} & \multirow{2}{*}{$\begin{array}{r}\text { frutas } \\
1999 \\
\end{array}$} \\
\hline & $1990-97$ & 1999 & $1990-97$ & 1999 & $1990-97$ & 1999 & & \\
\hline Australia & 300 & 344 & 95 & 96 & 116 & 213 & 103 & 105 \\
\hline Brunei & 1 & 0 & 39 & 44 & 5 & 6 & 46 & 27 \\
\hline Canadá & 189 & 163 & 116 & 138 & 168 & 180 & 59 & 22 \\
\hline Corea del Sur & 32 & 29 & 33 & 51 & 15 & 9 & 98 & 77 \\
\hline Chile & nd & 51 & nd & 99 & 96 & 55 & 134 & 191 \\
\hline China & 97 & 99 & 99 & 98 & & 88 & 101 & 100 \\
\hline China Hong Kong & 0 & 0 & 0 & 0 & nd & 0 & 16 & 1 \\
\hline China Taiwán & nd & 20 & nd & 16 & nd & 4 & 98 & 83 \\
\hline Estados Unidos & 135 & 134 & 99 & 101 & 141 & 138 & 97 & 81 \\
\hline Filipinas & 85 & 81 & 102 & 95 & 106 & 87 & 98 & 125 \\
\hline Indonesia & 91 & 86 & 117 & 108 & 98 & 95 & 94 & 102 \\
\hline Japón & 25 & 23 & 79 & 78 & 3 & 3 & 82 & 55 \\
\hline Malasia & 29 & 23 & 59 & 42 & 99 & 80 & 61 & 87 \\
\hline México & 79 & 71 & 91 & 85 & 41 & 26 & 146 & 111 \\
\hline Nueva Zelanda & 80 & 88 & 98 & 101 & 6 & 11 & 160 & 161 \\
\hline Papua Nva.Guinea & nd & 3 & nd & 100 & nd & 122 & 99 & 100 \\
\hline Perú & 43 & 52 & 99 & 98 & 92 & 72 & 108 & 99 \\
\hline Rusia & 90 & 88 & 100 & 98 & 115 & 151 & 92 & 53 \\
\hline Singapur & nd & nd & nd & nd & nd & nd & nd & nd \\
\hline Tailandia & 134 & 144 & 1046 & 697 & 95 & 77 & 114 & 122 \\
\hline Vietnam & 112 & 124 & 103 & 115 & 112 & 110 & 100 & 100 \\
\hline
\end{tabular}

compensan con los superávits de algunos países, en especial de los desarrollados (cuadro 3).

\section{Proteccionismo agrícola}

Por los elevados niveles de protección al sector agrícola, destacan en la región Japón, Corea y Taiwán. En el otro extremo están Nueva Zelanda y Australia, con una agricultura no protegida, mientras Estados Unidos y Canadá se encuentran en un grupo con niveles de protección intermedio (cuadro 4). En el caso de Japón, el nivel de protección medido por PSE, representó 65 por ciento entre 1986 y 1988 . Diez años después se ha mantenido cercano a ese nivel. Es decir que los apoyos otorgados al sector agrícola equivalen a cerca de dos terceras partes del valor de la producción agrícola. Corea presenta un caso similar. Ambos países se sitúan muy por arriba del promedio del PSE de los países de la OCDE (cuadro 4). En el otro extremo están Australia y Nueva Zelanda, en las que el último periodo el PSE se situaba en 6 y 1 por ciento respectivamente. En cuanto a Canadá y Estados Unidos se observa una tendencia a la baja en los niveles de protección, pero todavía son elevados; de 1996 a 1998, el PSE para Canadá era de 15 por ciento y para Estados Unidos de 17 por ciento. México, que ha emprendido un programa muy profundo de liberalización agrícola, mantiene un nivel de apoyo de alrededor de 14 por ciento y también presenta una tendencia a la baja.

Estos elevados niveles de apoyo, que pueden otorgarse vía precios elevados a los productores agrícolas por arriba de los precios internacionales y vía subsidios a la producción combinados con medidas de cierre de fronteras arancelarias o no arancelarias, se traducen al final en precios internos de los alimentos muy elevados y, paradójicamente, precios dumping fuera de los países exportadores. La medida utilizada para evaluar el impacto de los programas de apoyo agrícola sobre el consumidor, es el 'subsidio equivalente al 
consumidor' (CSE por sus siglas en inglés) que, cuando es negativo, implica una carga sobre el consumidor.

En Japón y Corea del Sur los elevados niveles de protección han tenido consecuencias tanto a nivel de sector como de la economía. A nivel de sector, ha inducido una asignación ineficiente de los recursos más escasos: tierra y mano de obra. A nivel de la economía, ha provocado que los precios de los alimentos que enfrentan los consumidores sean muy elevados comparados a los prevalecientes en los mercados internacionales. Peor aún, muchos estudios han señalado que las transferencias de los consumidores a los productores muchas veces se desvían en el proceso o bien llegan a los agricultores de mayores ingresos, a los menos necesitados (cuadro 4). ${ }^{14}$ Por tanto los países desarrollados son los que mantienen los más altos niveles de protección a sus sectores agrícolas, aun cuando ello les ha traído serias distorsiones en el uso de sus recursos y ha impactado de manera desfavorable el nivel de vida de los consumidores. Más aún, el impacto de las políticas proteccionistas no se circunscribe al país que las aplica, sino que tiene repercusiones más allá de sus fronteras. Los países con capacidad exportadora agrícola, por lo general países en desarrollo, ven frenadas sus exportaciones por la competencia de mercancías agrícolas subsidiadas en los mercados internacionales.

\section{Los resultados de la Ronda de Uruguay}

Por lo anterior, se propuso en la RU la eliminación de subsidios, la disminución de las tarifas, la eliminación de las cuotas y el mayor acceso a los mercados. En el 2000 se cumplió el plazo de aplicación de las medidas propuestas en la RU y aunque ha habido algunos avances, estos no han sido lo suficientemente importantes para reducir las ineficiencias en los mercados agrícolas internacionales. ¿Cuáles han sido hasta ahora los logros de la aplicación de los compromisos en materia agrícola en la RU? Entre los resultados más importantes esperados de la liberalización agrícola estaban, y todavía están, por eliminar la tendencia a la baja en los precios agrícolas y la extrema variabilidad en

Cuadro 4

APEC: Niveles de protección agrícola en países seleccionados (porcentajes)

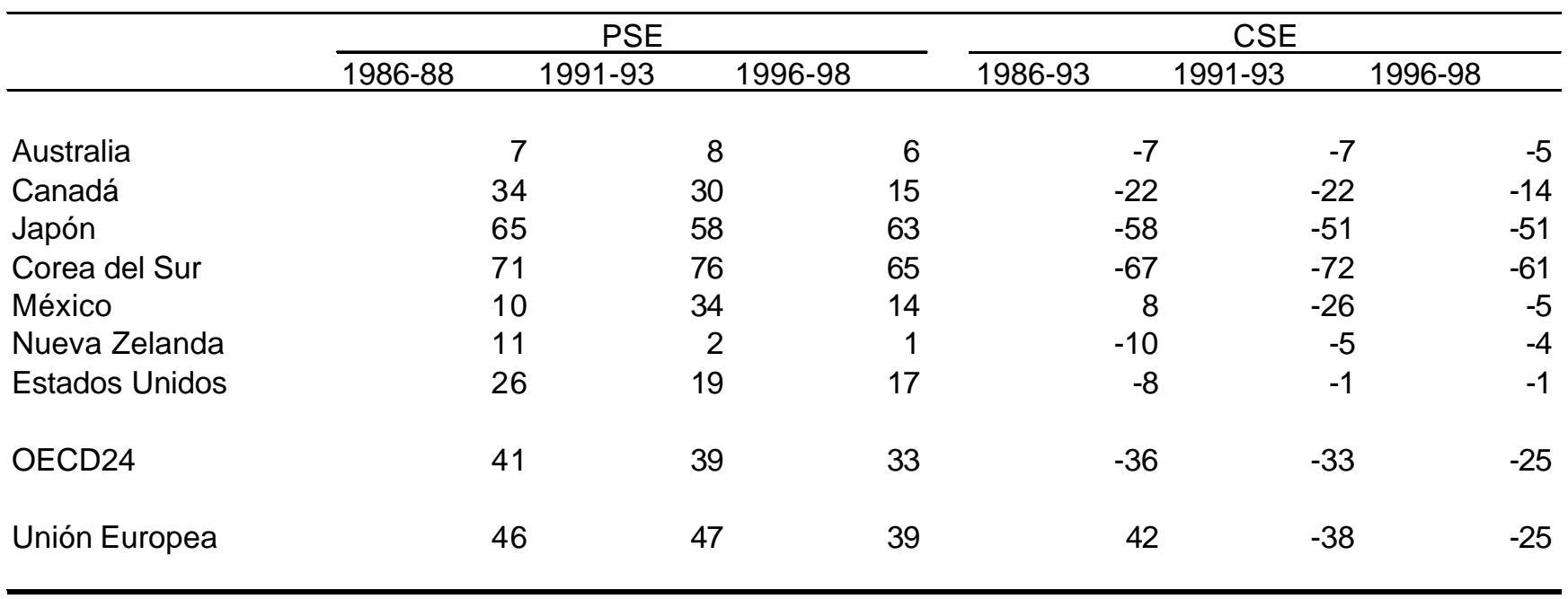

PSE=subsidio equivalente al productor CSE=subsidio equivalente al consumidor FUENTE: Agricultural Policies in OECD, Countries. Monitoring and Evaluation. 1999. OECD. 
los mismos. El proteccionismo de los países desarrollados, Estados Unidos, Japón y la Unión Europea, ha provocado que los precios agrícolas relativos a los precios de los productos manufacturados hayan tendido a descender, perjudicando con ello los ingresos de los productores de los países exportadores de estos productos, en su mayoría países en desarrollo. Con los apoyos a los precios de los productos agrícolas de los países industrializados, estos se vuelven superavitarios, y se deshacen de sus inventarios en los mercados internacionales a precios subsidiados. Ese exceso de oferta en los mercados internacionales deprimió a la larga los precios de los productos agrícolas.

Con la RU los países desarrollados se comprometieron a establecer "tarifas límites" equivalentes a la protección cuantitativa en el periodo 1986-1988, y a disminuir en promedio un 36 por ciento el nivel de protección hacia el año 2000 (para los países subdesarrollados los plazos fueron más largos y el ritmo de desgravación más lento). También se estableció el compromiso de disminuir los apoyos a la agricultura a un nivel equivalente al 80 por ciento del prevaleciente en el periodo 1986-88. Sin embargo, quedaron excluidos, como parte de los apoyos, los gastos contemplados en las cajas "azul y verde": los primeros relacionados con los apoyos para que los agricultores dejen de sembrar ciertos productos, y los segundos, relacionados con el medio ambiente. Lo que sucedió en la práctica fue que los países desarrollados aplicaron lo que se ha dado en llamar la "tarificación sucia". Esto significa que esos países fijaron sus tarifas límite a niveles muy elevados, por encima del nivel otorgado por la protección cuantitativa en el periodo base. Así, actualmente los niveles de protección arancelaria, incluyendo las reducciones programadas, son más altos que los prevalecientes en el periodo base. Aun cuando los países pueden establecer tarifas a niveles menores, tienen el poder discrecional de ajustarlas para "estabilizar el mercado" y ello mantiene la alta variabilidad observada en los precios agrícolas internacionales. ${ }^{15}$ Mientras las tarifas más elevadas en el sector manufacturero de los países desarrollados oscilan entre el $10 \mathrm{y}$ 12 por ciento, en el sector agrícola éstas llegan al 100 por ciento y en algunos casos alcanzan el 300 por ciento. Por otro lado el uso de barreras no arancelarias es muy frecuente en los países desarrollados. ${ }^{16}$

A pesar de que los sectores agrícolas de los países industrializados representan un porcentaje muy bajo del PIB, el fuerte apoyo otorgado a la agricultura se basa en las fuertes presiones de ciertos grupos de interés que constituyen una base electoral importante. Se ha calculado que en los ochenta las ganancias de los agricultores por las medidas proteccionistas en Europa, Estados Unidos y Japón, pasaron de 94 mil millones dólares a 141 mil millones de dólares, un aumento del 50 por ciento. Sin embargo, el costo para los consumidores, causado por las distorsiones en los mercados alimenticios, pasó de 120 a 216 mil millones de dólares, un incremento de casi el doble. Por otro lado, la riqueza de que disfrutan estos países les permite darse el lujo de financiar los elevados costos del apoyo, aunque sus políticas internas negativas han afectado los ingresos de los agricultores más pobres del mundo.

\section{La propuesta de un Sistema Alimentario en APEC}

Teniendo en cuenta la diversidad de condiciones materiales, financieras, políticas y sociales existentes en la región, las representaciones de las economías de APEC han planteado la necesidad de coordinar acciones en materia de comercio y producción agrícola. En este sentido, desde mediados del decenio pasado se propuso la creación de un sistema alimentario que considerara el comercio agrícola y de alimentos, a la par de atender los aspectos de transportación, finanzas, sustentabilidad del medio ambiente, cooperación económica, liberalización y facilitación, en beneficio de todos los miembros de APEC ${ }^{17}$. 
En la reunión de Vancouver de 1997, los líderes de las economías de la región dieron la bienvenida a la participación de empresarios académicos y otros expertos, en el foro de APEC; un año más tarde dieron instrucciones a sus ministros para que estudiaran la propuesta del Consejo Asesor Empresarial de APEC (ABAC), de establecer el 'sistema alimentario' de APEC (AFS). Partiendo de esta instrucción, en 1999 se estableció un grupo de trabajo adhoc para analizar dicha propuesta.

El sistema alimentario de APEC ha sido diseñado para cumplir con los objetivos más generales de ésta alcanzar en la región prosperidad económica, desarrollo sostenido y equitativo y seguridad nacional, teniendo como pilares el fomento de la liberalización y la cooperación técnica. Bajo una perspectiva de largo plazo se considera el impacto del crecimiento de la población y la expansión económica sobre los recursos energéticos, el medio ambiente y los alimentos. De ahí la necesidad de desarrollar una estrategia de cooperación entre los miembros de APEC para promover un desarrollo agrícola sustentable consistente con los principios de APEC de inclusión, no discriminación consistencia y flexibilidad con la Organización Mundial del Comercio (OMC). ${ }^{18}$

El AFS está previsto como un sólido proyecto de largo plazo que vincule eficientemente a los productores, los procesadores y los consumidores, de tal manera que asegure las necesidades futuras de alimentación de la región. El AFS incluye la colaboración pública y privada en tres áreas fundamentales: desarrollo de infraestructura rural, promoción del comercio alimentario y difusión de los avances técnicos, todo ello reconociendo las complejidades del sector agroalimentario en la región.

En la evaluación realizada por ABAC sobre el estado actual del sector alimentario en la región, se señala que la falta de políticas apropiadas está resultando muy costosa para la región, sobre todo para los países en desarrollo. Por otro lado, ABAC considera que los beneficios de cooperación en este sector serían potencialmente mayores que en cualquier otro. ABAC considera que los objetivos generales de APEC, de liberalizar en el 2010 y 2020 para países desarrollados y en desarrollo, debe permanecer. Desarrollar una agricultura sustentable, que considere el papel multifuncional de la agricultura, deberá alcanzarse con la colaboración de los países desarrollados, los subdesarrollados, los exportadores y los importadores. La propuesta no ha estado exenta de problemas. Mientras que las reformas a la agricultura continúan siendo una prioridad para las principales economías exportadoras (Estados Unidos, Australia y Nueva Zelanda), los países en desarrollo miembros del Grupo de Cairns (Indonesia, Malasia, Tailandia y Filipinas), y los países del noreste de Asia miembros de APEC (Japón y Corea del Sur) manifiestan serias reservas sobre el papel de APEC, respecto de las reformas a la agricultura.

El análisis de Gilbert, Scollay y $\operatorname{Sahl}^{19}$ a la propuesta del sistema alimentario de APEC realizado por medio de la simulación de un modelo de equilibrio general computable, concluye que, por una parte, los beneficios netos potenciales de la reforma agrícola en la región de APEC son substanciales y, por la otra, que la liberalización agrícola tendrá efectos negativos en los ingresos agrícolas en algunas economías. Ello ubica un problema político, que junto con otros aspectos importantes, como asegurar la seguridad alimentaria, deben ser considerados si se quieren realizar progresos significativos. La aplicación cuidadosa y selectiva de medidas de cooperación técnica y económica (Ecotech) consideran los autores- puede contribuir a mitigar los efectos adversos en el ingreso agrícola, esperados con la liberalización, y contrarrestar así la resistencia a la propuesta del AFS. Por medidas de Ecotech se entiende la promoción de actividades extra-agrícolas, la promoción educativa para el desarrollo de nuevas habilidades, transferencias de tecnología 
y políticas de desarrollo social como reforma agraria, programas de apoyo al aumento del ingreso y el nivel de vida de los agricultores, crédito, servicios de extensión y construcción de infraestructura.

Como se ha visto, en términos de nivel de ingreso y de dotación de recursos, la diversidad es muy grande en la región y por lo tanto el diseño de una estrategia de un sistema alimentario regional deberá tomarla en cuenta y establecer un balance muy delicado en la aplicación de las medidas, ya que éstas tendrán efectos muy diferentes sobre los actores involucrados. En países en los cuales el sector agrícola tiene todavía un peso relativo muy importante en términos de participación en la producción, en la generación de empleo y en las exportaciones, la liberalización del sector, propuesta por APEC, tendrá un efecto sobre la utilización de los recursos que deberá tomar en cuenta un periodo más largo de transición para el ajuste.

Dados los pobres resultados de la RU, la propuesta de APEC de crear un sistema alimentario en la región, deberá contar con la "buena voluntad" de los países desarrollados para revertir las tendencias en los mercados agrícolas, ya que en el caso de la región, Estados Unidos es el jugador más importante. No obstante, aún en casos como el del Tratado de Libre Comercio de América del Norte (TLCAN), bajo el cual las partes están comprometidas a alcanzar metas específicas de liberalización, Estados Unidos se ha mostrado renuente en varias ocasiones a cumplir con sus compromisos. Es el caso del jitomate, el atún, el aguacate y, recientemente, el transporte, y ello ocurre bajo las restricciones de un acuerdo formal entre los tres países. El regionalismo de APEC es abierto y flexible, lo que implica menor compromiso de las partes. Los países en desarrollo han hecho o están haciendo su parte, se han liberalizado unilateralmente; los desarrollados tienen que mostrar un mayor compromiso.

\section{Conclusiones}

$\mathrm{Al}$ analizar las políticas y la participación de los diferentes actores en la región de APEC, queda claro que el objetivo de la propuesta de $\mathrm{ABAC}$ de crear un sistema alimentario en la región es muy difícil y complejo de alcanzar. Es necesario que los países con altos niveles de protección, como Estados Unidos, Japón, Corea del Sur y Taiwán, se comprometan seriamente a abrir sus mercados para brindar oportunidades a los exportadores netos de la región. Tanto ASEAN4, como China, Nueva Zelanda y Australia se beneficiarían de esta apertura. Para los menos desarrollados ello significa poder consolidar un desarrollo exportador agrícola que les permita importar los bienes para su crecimiento industrial.

Los casos de México y Filipinas, que se encuentran entre los importadores netos de la región, reflejan una pérdida de oportunidad en los mercados de la zona. Ello quiere decir que tienen que aplicar estrategias agrícolas integrales que permitan crecer a sus sectores y que puedan insertarse de una manera más eficiente en los mercados agrícolas.

En los casos de los exportadores netos menos desarrollados, ASEAN-4, Vietnam y China, todavía existe un potencial por desarrollar dados sus bajos niveles de productividad agrícola. Sin embargo, el impulso agrícola deberá llevarse a cabo con una menor degradación del medio ambiente, que en algunos países como Tailandia es un problema serio. De ahí que el objetivo del proyecto FAS de lograr un desarrollo sustentable sea prioritario, así como el de la cooperación en la difusión de técnicas avanzadas en la agricultura. Japón, por ejemplo, tiene un amplio desarrollo en biotecnología y otras áreas relacionadas, y debería proporcionar más ayuda de la que ya proporciona a través de sus agencias de cooperación, como la Japan International Cooperation Agency (JICA), o Estados Unidos a través de Agencia Internacional para el Desarrollo, AID. 
En cuanto al objetivo de liberalización de los mercados agrícolas contenido en la propuesta AFS, este se presenta como un problema difícil, si se toman en cuenta los pobres resultados obtenidos en la Ronda Uruguay. Los países proteccionistas desarrollados de la región deben comprometerse a eliminar sus altos niveles de protección y brindar con ello mayores oportunidades a los productores agrícolas de otros países menos desarrollados, de lo contrario, el futuro se torna pesimista si se quiere lograr un verdadero sistema alimentario en la zona en la cual los países compitan bajo condiciones de igualdad. Sólo así podrá llevarse a cabo un desarrollo sustentable y equitativo en la región, donde el papel de China será crucial. Su aceptación en la OMC traerá beneficios a todos $\mathrm{y}$, junto con un mayor nivel de ingreso, las importaciones de alimentos de este país tenderán a crecer, lo que dependerá también de la apertura que se dé a los mercados de textiles y ropa, en los cuales tiene ventaja comparativa. Si ello sucede, más recursos tendrán que fluir hacia esos sectores procedentes del agrícola, por lo que China deberá incrementar sus niveles de productividad en el sector para alimentar a una quinta parte de la población y deberá complementar esta con mayores importaciones. Con voluntad puede lograrse un balance regional alimentario, pero hasta ahora este no ha sido el panorama.

\section{Notas}

1 Estas cifras provienen de la FAO y en ellas no se incluyen las importaciones de productos marinos, lo cual subestima la participación relativa de la región.

2 Sobre agricultura en el Pacífico, véase también Rae, Allan, ed., Pacific Rim Agriculture, Opportunities, Competitiveness and Reforms, PECC, Centre for Agricultural Policy Studies, Massey University, New Zealand, April 1993 y Coyle, William, Hayes, Dermot and Yamauchi, Hiroshi, Eds., Agriculture and Trade in the Pacific, Toward the Twenty-First Century, Westview Press, Boulder, USA, 1992 y Anderson, Kym, Dimaranan, Bettina, Hertel, Tom and Martin, William, "Asia-Pacific Food Markets and Trade in 2005: A Global, Economy-wide Perspective". Presentado en la conference on Food and Agricultural Policy Challenges for the Asia Pacific, Manila, Filipinas, octubre 1996.

3 OECD, Agricultural Policies in OECD Countries, Monitoring and Evaluation, OECD, 1999, pp. 148-152.

4 Center for International Trade Expansion "U.S. Food and Agricultural Policies. 2996 Farm Bill" (http://www. ssu.missouri.edu [28/10/00]); Ontario, Ministry of Agriculture, Food and Rural Affairs, "1996 U.S. Farm Bill Analysis” (http://www. gov.on.ca.[16/09/00]).

5 El PSE mide el monto total de los programas de apoyo al sector, como proporción del valor de la producción. Así, un PSE de $17 \%$ significa que el monto del apoyo al sector representa $17 \%$ del valor de toda la producción agrícola.

6 Agriculture and Agri-Food Canada. All about Canada Agri-food Industry. (wysiwyg://8/ http://aceis.agr.ca/cb/ factsheets/2indus-e.html). Consulta: 9/07/00.

7 Pacific Economic Cooperation Council (2000), Pacific Food Outlook, 1999-2000.

8 Found, C. William ( 1999), "Agriculture in a World of Subsidies", en Britton N.H. Jonh (Edit.), Canada and the Global Economy. The Geography of Structural and Technological Change, McGill-Orcens University Press, Montreal, pp. 155-168

$9 \quad$ Ibid, p. 157

10 Los datos de la FAO (FAOSTAT, Database), confirman los planteamientos que a este respecto hicieran Kym, Dimaranan, Bettina, Hertel, Tom and, Martin, Will, en su ponencia "Asia-Pacific Food Markets and Trade in 2005: A Global, Economy-wide Perspective", Conference on Food and Agricultural Policy Challenges for the Asia Pacific, October 1-3, 1993, Manila. (Inédito).

11 Anderson, et al., op. cit.

12 Que, Tran Thi, "Economic Reforms and Their Impact on Agricutural Development in Vietnam" en Asean Economic Bulletin, Vol. 15, Núm. 1, April 1998, Special Focus: Development Strategies, Agricultural Policies and Agricultural Development in southeast Asia, Ed. Than, Mya.

13 Ibidem. (Este número de el Asean Economic Bulletin contiene varios artículos sobre la agricultura en los cuatro países incluidos en Asean4).

14 Véase, Anderson, Kym y Tyers, Rodney, Global Effects of Liberalizing Trade in Farm Products, Trade Policy Research Center, University of Michigan Press, Ann Arbor, USA, 1991.

15 Anderson, Kym, "Agriculture, WTO, and the Next Round of Multilateral Trade Negotiations", Center for International Economic Studies, University of Adelaide, Australia, November 1998 de internet, http:// www.cairnsgroupfarmers.org [25/05/99].

16 Stoeckel, Andy, Vincent, David and McKibbin, Warwick, "Global Trade Reform, Mantaining Momentum, Center for International Economics", Australia, 1999, de internet, $\underline{\text { http:// }}$ www.cairnsgroupfarmers.org [25/05/99].

17 U.S. Nacional Center for APEC "Proposal for a Pacific Rim Food System" (www2. Hawaii.edu/apfat/iep5.htm.[ 25/05/99]).

18 Véase APEC, "Apec Food System. A Study by an Adhoc Task Fforce of the Ssenior Officials Meetin" on An Apec Food System Proposal from the Apec Business Advisory Council, April 19, 1999.

19 Gilbert, John, Scollay Robert and Wahl Thomas, "The APEC Food System: Implication for Agricultural and Rural Development Policy", The Developing Economies, XXXVII-3, September 2000, pp. 308-329; The World Economy, Oxford, febrero de 2000. 